\title{
A Primer on Practical Significance
}

\author{
Miguel Alejandro A. Silan \\ University of the Philippines Diliman
}

\begin{abstract}
One of the main criticisms of Null Hypothesis Significance Testing (NHST) is that statistical significance is not practical significance. And this evaluation of the practical significance of effects often take an implicit but consequential form in the field: from informal conversations among researchers when evaluating findings, to peer reviewers deciding the importance of an article. This primer seeks to make explicit what we mean when we talk about practical significance, organize what we know of it, and assert a framework for how we can evaluate and establish it. The practical significance of effects is appraised by analyzing (i.) along different levels of analysis, (ii.) across different outcomes, (iii.) across time and (iv.) across relevant moderators; which also underlie the conditions of when small effect sizes can be consequential. Practical significance is contrasted with often conflated terms including statistical significance, effect size and effect size benchmarks as well as theoretical significance. Promising directions are then presented.
\end{abstract}

Keywords: practical significance, effect size, effect size benchmark, meta-science, meta-methodology

Say a study between two randomized groups was done to test the intergroup contact hypothesis - the treatment group of straight cisgender individuals were made to have a conversation with trans individuals, and the placebo group was made to have a conversation with fellow straight cisgender individuals, after which both answer an 8-item attitude towards trans individual scale. If the study found a Cohen's $d=0.39,95 \%$ CI $(0.231,0.554)$ how do we interpret these indices beyond just the mere magnitude of their group mean difference and the overlap across the two groups?

Over the recent years, methodological reforms have fast progressed, with advocates correcting widely misinterpreted and mindless use of p-values and common Frequentist analysis (Lakens, 2016; Mayo, 2018), pushing to utilize effect sizes and uncertainty bounds (Cumming, 2013; Kline, 2004) and Bayesian procedures (Kruschke, 2014), along with concomitant push for open science (Chambers, 2017), structural reforms, as well as advocating for better training (Munafò, et al., 2017), better measurement (Flake, Pek, \& Hehman, 2017) and better theory (Fiedler, 2018; Borsboom, 2013)

This article seeks to add to this discussion, particularly of the first two: research articles that report statistical significance and effect size estimates should also routinely (try) to establish practical significance. While there is an attempt to be general, this article is written in the locus of social psychology, and thus harks on its norms, standard ways of analysis, research culture and so on. And this is especially written for pragmatists (Fried, 2017) and applied oriented psychologists who frequently want to see the usefulness of various results, research reports, constructs and theories across domains in psychology

Across these various fields, the calls to establish practical significance is not new (e.g. Yeaton \& Sechrest, 1981; Kirk, 1996; Aguinis et al., 2010). This is usually discussed as a counterpoint to what statistical significance can and cannot answer. Baguley (2012) for example state that the "final point is that NHSTs cannot, in isolation, indicate how important, large or useful an effect is. The $p$ value has a very narrow interpretation as the conditional probability of a statistic that is as extreme (or more extreme) than that observed if $\mathrm{HO}$ is true." Advocates of effect size estimation have used the same rhetoric, where some attack statistical significance as limited, or worse, misleading (e.g Kirk, 1996; Cumming, 2014). However, statistical significance testing when done right is a valuable and powerful tool that allows researchers to decide when to rule out sampling variability as the cause of differences seen in data, which allows to rule out canonical errors (Mayo, 1996) and improving inference (Lakens, 2016; Lakens, 2017). Effect size estimation, meanwhile, allows one to answer the additional question of how large the size of the effect is (and its corresponding uncertainty by confidence or credibility intervals). However, a practical significance approach takes this one step further.

The difference of these approaches (statistical significance, effect size and practical significance) seem to take an implicit but not inconsequential form in the 
field: whether as informal conversations between researchers when evaluating findings, or peer reviewers deciding the importance of an article. Thus, this primer seeks to make explicit what we mean when we talk about practical significance, organize what we know of it, and assert a framework for how we can evaluate and establish it.

\section{Practical Significance through the ages}

Many of the earlier articles operationalizing practical significance (especially as contrasted to statistical significance) come from the field of medicine and clinical psychology (e.g. Jacobson \& Truax, 1991; Lydick \& Epstein, 1993; Osoba, Rodrigues, Myles, Zee \& Pater, 1998; McGlinchey, Atkins, \& Jacobson, 2002) where presumably, the accessibility of both quantitative and qualitative information allows better calibration of what statistical significance of the data mean, and whether it converges with qualitative outcomes. Speculatively, it seems that clinicians administering a scale will also observe and interview the patient in the course of therapy, wherein social psychology researchers typically do not or cannot identify their sample again after validating a scale or administering an experiment. Further, it seems that given the importance of knowing the efficacy of treatments, there is greater pressure to more clearly explain what effect sizes mean in non-arbitrary units such as life-years gained, decrease in probability of getting the disease and so on (Guyatt, Rennie et al., 2002)

However, writings about practical significance through the decades have a messy nomenclature. Early writers (Kirk, 1996; Steyn, 2000; Ellis \& Steyn, 2003; Onwuegbuzie \& Levin, 2003) equate effect size measures to be direct indicators of practical significance, however as will be discussed below, we disagree. Further, some distinguish between effect-size-aspractical-significance and clinical significance (e.g. Thompson, 2002: Pintea, 2010). In this article, clinical significance is just practical significance in clinical setting, and are not two inherently different constructs. ${ }^{1}$ Thus, practical significance here is much closer to how Vaske, Gliner \& Morgan (2002), as well as Plonsky \& Oswald (2014) and Ferguson (2009) used the term..

\section{WHAT IS PRACTICAL SIGNIFICANCE?}

Statistical significance typically asks whether an effect is non-null, effect size quantifies the magnitude of this effect, but practical significance is whether this quantified, non-null effect seen in data meaningfully changes something in the world

Thus, we define practical significance as how effects -quantified by effect size measures found in studies (say $r=.67, d=0.45, b=0.2$ etc.)- correspond to day-to-day behaviors and other meaningful social outcomes.

We focus here on effects 'operationalized' through effect size (ES) measures rather than constructs, theories, other latent variables not because we are not interested in the latter, but to emphasize that practical significance is different from theoretical significance and the evaluation of the two will not necessarily be similar. Further ES measures are 'agnostic' and pragmatic: it may fit competing theoretical models and data generating processes, but any given ES measures considering a study's design, sample and results can be subjected to an evaluation of practical significance. [See assumptions and philosophical debates about effect sizes below]. In this framework, whether a given Extraversion $E$ is due to within-person network processes or whether it's a bio-social-psychological causal latent variable (Cramer et al., 2012) matters less than if individual $i$ with $E$ actually goes to parties, have more frequent social interactions, feels more energetic after socializing etc.

These effect sizes include the 'd-family' measures, including Cohen's $\mathrm{d}$ and its bias-corrected estimates like Hedge's g. [For a review, see Cumming, 2013; and for considerations in which effect size measure to use: Onwuegbuzie \& Levin, 2003] As well as the 'r-family' measures including Pearson's $r$, variance explained measures like $r^{2}$ and ANOVA's $\eta 2$ and $\eta p 2$ and its bias corrected estimate, $\omega 2$ and $\omega \mathrm{p} 2 \mathrm{We}$ also include here $b$ parameters of SEM (e.g does the change in latent variable $\mathrm{V}$, affect day-today family behavior $\mathrm{Y}$ ?), interaction terms, simple difference-in-means, simple differences-in-differences as well as other measures of average treatment effects in experiments (see: Gerber
1 There is a stream of clinical significance literature that define clinical significance as the change in patients' score after treatment, with cutoffs being decided through different formulas (the typical one being the Jacobson-Truax Method and its variants) (Lamber, Hansen \& Bauer, 2008; Jacobson \& Truax, 1991). This and other conceptualizations of clinical significance; including that treated patients should be empirically indistinguishable from 'normal' peers, and subjective perception of client and therapist can be subsumed under the general framework presented in this paper. 
$\&$ Green, 2012). Although we note the limits of groupto-individual generalizability in effect size measures. ${ }^{2}$

Following the definition above, a more limited, but useful framing can assert that practical significance is how quantitative metrics correspond to qualitative behaviors or outcomes. This is particularly important for when the measurement units are arbitrary [see discussion of arbitrary measurement in the next section]. However, when meaningful, non-arbitrary units are used (e.g., death/living, voted/not voted, the systematic observation of behavior such as the frequency of day-to-day absenteeism etc.) then the business of the practical significance approach is to make the effects -its magnitude and uncertaintyinterpretable. Without looking at meaningful realworld outcomes, effect sizes measures of any size cannot be determined to be practical.

For example, if a prejudice reduction intervention in college decreases the group average score in a prejudice scale, against a control group (significant at alpha 0.05 , with $\mathrm{p}=0.002, \mathrm{~d}=0.32$, CI: $0.29-45$ ) does this mean that members in group $\mathrm{A}$, on average, will now more frequently interact with minority group members? The interactions warmer? More willing toand actually voting for minority beneficial policies? And will minority members perceive him/her in a better light? Statistical significance tells us the underlying effect parameter is not likely to be zero, the effect size gives a summary taking into account the difference of means and their pooled variability, but the practical significance is undetermined and is yet to be established.

Further, there will always be arguments about the 'meaningfulness' of an outcome (Guyatt, Osoba et al., 2002), first of which is to ask, 'meaningful for who?' the study participants? The researchers? Policymakers or other stakeholders? To disentangle this, and make 'meaningfulness' less abstract, we can analyze (i.) along different levels of analysis, (ii.) across different

\footnotetext{
2 Certain effect size estimates, such as correlation and other statistical measures that use this as the basis, such as factor structure when derived from a cross-sectional sample cannot be assured to have same values for longitudinal samples (Molenaar, 2009). This lack of group-to-individual generalizability feature heavily on within human processes such as personality, intelligence, cognitive processes, and mental disorders; so estimating these from cross-sectional samples are said to have potentially misleading estimates and interpretation (Borsboom, Kievit, Cervone, \& Hood, 2009; Gomes, de Araujo, do Nascimento, \& Jelihovschi, 2018)
}

However, this is not the case for experimental studies, in which the average treatment effect is typically the best guess for any particular individual's treatment effect (Gerber and Green, 2012). How outcomes, (iii.) across time and (iv.) across relevant moderators:

\section{Practical significance can be evaluated by} looking across different levels of social organization, including: (a.) "subjective", (b.) individual, (c.) groups, including families, work units, organizations as well as social networks both online and offline and (d.) economy/society; or the interacting collection of the above stated units. Practical significance may or may not correspond across these levels. That is, an effect in one can, but doesn't necessarily translate to an effect in other levels.

For example, training interventions may increase at the individual-level the awareness of the benefits of diversity, but not necessarily affect higher level hiring practices of an organization (Kulik \& Roberson, 2008). A "green policy default" on energy tariffs might substantially reduce carbon dioxide emission at the municipal level (Pichert \& Katsikopolous, 2008) but the reduction of environmental damage may not be perceived on average by any single individual in the region. As Rothman (2004) asserts "interventions can have minimal impact on an individual's behavior but when disseminated widely have a dramatic impact at the societal level". However, it may very likely correspond: a family clinical intervention might promote stronger family bonds and open communication and promote individual functioning and reduce distress (Szapocznik, \& Williams, 2000; Stoddart, 1999).

We differ here "subjective" and individual practical significance in the knowledge that effect size measures might correspond to objectively different outcomes for an individual but which may not necessarily be subjectively appreciated by the individual; for example actual versus feelings of learning when encountering a new topic (Deslauriers, McCarty, Miller, Callaghan, \& Kestin, 2019). On the flipside, an individual may subjectively feel different, as in being more confident

close this is to any ones' score depends on the distribution of treatment effects (ex. if it has more than one mode); various types of moderators might be included in the model to refine the guess for an individual's extrapolated estimate (i.e whether women are more receptive to a particular smoking prevention campaign, or whether low achieving students benefit more from a revised educational curriculum). However, the variances of the mean for cross-sectional and longitudinal data may differ quite largely, especially for small sample sizes (Fisher, Medaglia, \& Jeronimus, 2018)

Put another way, associative designs necessarily use interindividual ranking to produce a particular statistic. A change of $n=500$ to $\mathrm{n}=1$ makes a correlation non-existent (Borsboom et al., 2009) but makes the calculated average treatment effect simply particularly sharp: the individual's own treatment effect 
after 'power posing', despite the lack of change of other objective metrics (Cuddy, Schultz \& Fosse, 2018; Credé, \& Phillips, 2017; Credé, 2018). Thus, placebo studies can obtain subjective practical significance despite the lack of effects on other levels or any other outcomes.

Another interest here is the evaluation of effectsproduced-through-interventions on their economic significance, usually framed through the level of society-at-large (Leech \& Onwuegbuzie, 2004). This can be evaluated through different indices, including: (i.) Cost Effectiveness, (ii.) Cost Benefit, (iii.) Cost Utility, (iv.) Cost Feasibility and (v.) Cost Sensitivity. Leech \& Onweugbuzie's example (2004; from Lewis et al. 2003) is training teachers in a new block schedule in high school. This shifts traditional high school schedule, where each class has around 45 minutes per day to a new scheme where there are fewer classes but with longer 90 minute durations. In this new schedule, low achieving students seem to have a statistically significantly higher test scores (Mean diff $=12.81, \mathrm{SE}$ of mean diff $=2.34$, $\mathrm{df}=83, \mathrm{p}<0.001, \mathrm{~d}=1.20$ ). If the shift costs $\$ 300,000$ then the cost effectiveness index is $(\$ 300,000 /$ mean diff $)=\$ 23,310.02$. This represents that each one-point difference in mean scores cost around $\$ 23,310.02$. Its cost benefit index meanwhile, requires the computation of the benefit (including nonmonetary benefits such as improved attitudes to learning) and subtracting this from the program cost. Cost utility on the other hand, requires the calculation of utility; which necessarily involve the qualitative approach of asking each group of stakeholders involved (including the high school students who are participants of the intervention) to decide on the values of each outcome. This index then gives us the cost of obtaining a single unit of utility (see also Cabrera \& Raju, 2001). Cost feasibility determines whether the cost of the intervention is within a set budget, and cost sensitivity represent the uncertainty involved in the calculation of the previous indices.

\section{Further, practical significance can also be} evaluated by looking across different outcomes. For example, a particular educational-entertainment radio program in a developing country may change perceived norms, increase willingness to speak out on difficult subjects and change the dynamics of community negotiations, but not willingness to affiliate with other tribes nor change beliefs about violence and other related attitudes (Paluck, 2009). Or like before, a therapy may positively affect a wide range of outcomes: occupational functioning, relationship with friends, relationship with family and self-reported well- being (Stoddart, 1999). Thus, when establishing and evaluating the practical significance of effects, it is important to consider a wide range of outcomes.

Linking with the discussion above, a given effect or an intervention might affect different outcomes across different levels of organization. The therapy for depression that promotes individual patient functionality might be linked to the patient's family's distress due to the cost of therapy (Mohr et al., 2006; Giuffrida, \& Torgerson, 1997; Nock, \& Ferriter, 2005). And the previously discussed high school block schedule (e.g Leech \& Onwuegbuzie, 2004) might have practical effects on the subjective and individual level (i.e that students on average, feels like they learn better and do get slightly better grades) but introducing the new schedule for school teachers may cost a lot more than the monetary equivalent of its benefits. On the other hand, an intervention, such as the development of an effective and valid organizational selection process (Rumsey, 2012) may likely positively affect outcomes across a wide range of levels, from individual to group to economic. A valid selection tool may likely foster better work-individual fit and more coherent units both of which result to more cost-effective ways to tackle the organizational goals

III. Practical significance should also be seen in the temporal nature of effects. An intervention might have an immediate effect but dissipate later. It may also affect outcomes both immediately after, and sometime later; the latter of which can be because (i.) the effect persists due of the durability of the phenomena. Learning how to vote by modelling parents' voting behaviors will theoretically not diminish the learning itself through time; whether the for a 2 nd time voter or a 5th time voter, despite the probability of voting itself decreasing over time (Gidengil, Wass \& Valaste, 2016). (ii.) the effect persists because of repeated production/administration of the treatment/stimuli, such as the case for marketing to increase product sales (e.g Pechmann \& Stewart, 1988; Johnson, Lewis, \& Reiley, 2016) (iii.) The effect may also persist because the intervention have downstream consequences (Gerber \& Green, 2012). For example, education at an elite university need not be durable or repeatedly administered to an individual to produce downstream effects such as higher earnings and occupational achievement (Hoekstra, 2009; Brand \& Halaby, 2006) (iv.) Further, direct and indirect effects can cause changes in outcomes across time. For example, grandparents can have an indirect influence on grandchildren's financial literacy by being the model of the parent's financial behaviors which in turn are 
modeled by the grandchildren, and through direct conversations with grandchildren about financial planning. (LeBaron, Hill, Rosa \& Marks, 2018)

However, effects may also not be immediately noticeable, but have a "sleeper effect". Early formulation of attitude inoculation -resistance to counterattitudinal arguments due to initial presentation of weak arguments- predict that a delay is needed so individuals can form arguments that defend their positions. (McGuire, 1964) however evidence do not support this formulation (Banas \& Rains, 2010). Any 'sleeper effects' might not be common in social psychology and will most likely be better characterized as latent learning. However, this possibility is not discounted (for example in processes of minority influence; Butera, Falomir-Pichastor, Mugny, Quiamzade, 2017) The nature of temporal dynamics of effects, both in within-person processes (Borsboom, 2017; Cramer et al., 2012) as well as in social networks (Chan \& Fu, 2015; Shalizi \& Thomas, 2011) remain an area of active study

IV. Practical significance should be assessed by how effects differ across moderators. These moderators include (Kline, 2008): (i.) treatment-byunit interaction, whether the effect holds across different units with different characteristics, (ii.) treatment-by-setting interaction, whether the effect holds across different settings, (iii.) treatment-bytreatment interaction, whether the effect holds across varying intensity of the treatment or its level of 'dosages', (iii.) multiple treatment interference, whether the effect of one variable on our outcomes of interest will hold in the presence of other 'interfering' variables. That is typical laboratory experiments show what "can affect people's behavior without determining whether, in a more complex setting, they do affect behavior." (Rothman, 2004; Mook, 1983). A principled study of the effects of moderators are can be facilitated done through large-scale collaborations across multiple lab sites (Klein et al., 2018; Moshontz et al., 2018). However, this is not to encourage the use of 'hidden moderators' to defend hypotheses that do not replicate [See section on assumptions].

This section demands that we ask, "effects are practically significant for who?" Particular pedagogical techniques for example might be empirically shown to affect student learning, but a proper moderation study would show that these increase scores of already high-

\footnotetext{
${ }^{3}$ Promising directions include work by Imai and Ratkovic (2013) on differential causal effects on subgroups. For other quantitative handling of practical significance, promising tools include multilevel and multivariate related outcomes models (e.g. Baldwin, Imel,
}

achieving students anyway. ${ }^{3} \quad$ Therefore, unlike Neyman-Pearson statistical significance with clear decision rules (accept-reject), practical significance can be seen as a high-dimensional concept, which is better handled as a heuristic and the establishment of which requires it to be well qualified: the effect is linked to practical concrete outcome/s, in a concrete level/s, in a concrete time, in a concrete unit.

Hence, this paper deals with practical significance heuristically ("how does an effect, represented by effect size measures and test statistics, correspond to meaningful behaviors?"), rather than formally. No attempt is made to formalize this into a single, global "practical significance score". However, given the state of the field, we believe there is no rush to create quick summaries.

As further discussed below it is up to the scientistpractitioner (or the pragmatic psychologist) to show that substantive and statistical assumptions of different research designs are met (ex. Non-confounding $n$ experiments, measurement validity and reliability in observational studies)

\section{WHAT IS PRACTICAL SIGNFICANCE NOT?}

Effect Size estimates are not practical significance. Prior literature sometimes argue that effect size estimates are themselves indicators of practical significance (e.g. Kirk, 1996; Steyn, 2000; Ellis \& Steyn, 2003; Onwuegbuzie \& Levin, 2003). However, as discussed, effect sizes typically answer a different question to practical significance; and the two only asks a similar question if the units used are non-arbitrary. Grissom and Kim (2005) best represent this position when they state that although "[effect size] is not synonymous with practical significance, knowledge of a result's effect size can inform a judgment about practical significance."

While large effect sizes are more likely to be practically significant (Pintea, 2010) small effect sizes -as long as they are reliable- can be practically significant especially if

1.) They affect higher levels of social organization. Small effects for any one individual will remain small effects, but 'small effect size measures' or small changes in how organizations are run and what laws are enacted, represent small effects across individuals in a whole swathe of population.

Braithwaite, \& Atkins, 2014) and other developments in causal inference (e.g Cunningham, 2018) 
However, as with all these guidelines, a rigorous evaluation of the phenomena, study or effect must still be undertaken to prove the actual practical significance. Behavioral economic nudges for example without a formal cost-effectiveness analysis, is just assumed to be a cost-effective measure when equally costly but more effective alternatives may exist (Benartzi et al., 2017)

2.) They affect a wide range of outcomes. An individual's cognitive ability, SES and personality for example affect a wide variety of consequential outcomes across the lifespan, including occupational attainment, divorce and mortality (Roberts, Kuncel, Shiner, Caspi, \& Goldberg, 2007). Unlike an intervention -which affects a group of subjects in a given time- personality, SES and cognitive ability/intelligence is not just carried across the various situations the individual faces in one's day-to-day life (including educational opportunities, occupational opportunities, exposure to particular social networks etc.) but also, across time in one's life. Despite having a 'small effect' on any single situation, because it affects such a broad range of settings and outcomes, the effect on the individual is highly practically significant.

3.) They do not dissipate over time, and/or accumulates (Cortina \& Landis, 2009; Yeaton \& Sechrest, 1981). Advertising for example, deal with effect that easily dissipate at least in terms of attention, if not recall. But because of repeated exposure, a small effect size on any particular individual can still lead to huge revenue streams (Pechmann \& Stewart, 1988; Johnson, Lewis, \& Reiley, 2016). Lise Eliot (2010) also detail how boy-girl expectations can be small in any single setting such as when parents stopping girls from crawling a steep incline earlier than boys, or are more open with their communication of feelings with girls than with boys etc. However, these 'small' expectations and reinforcements lead to noticeable but non-innate gender differences in adults. Consequently, a multitude of small, but consistent effects have practical consequences on gender differences between verbal ability, activity level, aggression and emotional expression for adult males and females, especially in U.S. individuals (Eliot, 2010)

4.) If the factor causing the effect is manipulable (Matz, Gladstone, \& Stillwell, 2017). Especially if it benefits more than it costs, if the outcomes are consequential, and if the change is done at a higher social level. For example, relationships, personality and stable employment are all stronger predictors of well-being than consumption choices. However, of these, only the latter are easily changed by the individual. So even if it is "negligible for a single consumer, but if a retail giant like Amazon aimed at making its customers happier by personalizing their product recommendations, a $1 \%$ increase in life satisfaction across its 244 million customers (Kline, 2014) could turn a small effect size into a huge social effect." (Matz et al., 2017)

5.) They affect outcomes that are hard to change (Prentice \& Miller, 1992; Ferguson, 2009). if the effects themselves change outcomes that are traditionally or empirically seen to be difficult to change -such as personality, intractable conflict and poverty- and does so reliably; then any small effect size can be said to be meaningful.

It's also noted that large effect size measures if chosen poorly has the potential to be misleading. Jacobson and Truax (1991) for example assert that "if a treatment for obesity results in a mean weight loss of $2 \mathrm{lb}$ and if subjects in a control group average zero weight loss, the effect size could be quite large if variability within the groups were low. Yet the large effect size would not render the results any less trivial from a clinical standpoint." That is, it may affect the outcome of weight loss, possibly positively affect the outcome of subjective significance, but not the change in risk of serious illness, life-years gained and so on.

Effect size benchmarks are not indicators of practical significance. Given the recent focus on effect size estimation, many also forward the more routine use of domain specific effect size benchmarks (Bosco et al., 2015) as a better alternative to the popular age old benchmarks of Cohen (in Cohen, $1962|\mathrm{r}|=.2, .4$ and .6 was written as small, medium and large respectively; which was revised in Cohen, 1988 in the now iconic $|r|=.1, .3, .5$.) But Cohen himself notes these guidelines should give way to domain specific benchmarks if there were any.

Like effect size estimates, effect size benchmarks are not indicators of practical significance. Tremendous effort is made to map these domain specific benchmarks (e.g Plonsky \& Oswald, 2014; Paterson, Harms, Steel \& Crede 2015; Bosco et al., 2015), and they are useful guides of how an effect compares to other studies in the same domain. That is, they answer best the question of the typicality of a reported effect size of one study compared to the field. However, if in this network of studies, none tap behaviors and other meaningful outcomes; then it may become misleading. It may tempt the researcher to provide an answer to a question not asked by the method ("how does the size compare to a group of studies" vs "is the study practically significant"). As Ulric Neisser (1974) advocates, somewhere along the line, we must touch base with reality. 
Further, the linking effect sizes to practical outcomes help in creating interpretable benchmarks for effect size values, and answering how large is large enough to matter ${ }^{4}$.

Moreover, practical significance as defined here is not incompatible with Lakens' (2017) 'smallest effect size of interest' (SESOI). While SESOI is an a priori expectation or decision, the practical significance approach here is the establishment of the correspondence between effect size measures and meaningful outcomes and behaviors. It then helps in aiding researchers calibrate the SESOI, which in the first place is difficult to construct without knowing what the ES measures represent in non-arbitrary terms.

Statistical significance and practical significance. There has been a long list flogging p-values, repeatedly establishing that statistical significance is not practical significance. It obviously is not, but it doesn't mean that hypothesis testing is any less valuable in a methodologists and pragmatic psychologists' arsenal. It has been argued that effects can be practically significant without being statistically significant and vice versa (e.g. Thompson, 2002) this position is difficult to agree with entirely. Without hypothesis testing, or other ways to rule out sampling variability (such as replication) effect sizes can be misleading. Accordingly, principled use of Frequentist statistics (i.e. what p-values across studies do we get if the effect of interest exists, given certain study parameters? and what claims are warranted if they pass severe tests?) can be an important step before evaluating practical significance, especially in making sure that an effect exists in the first place.

\section{Validity isn't necessarily practical significance}

Scales and measurement in experimental settings can be valid in that it measures what it intends to measure, but validity is a not a guarantee that a scale/phenomenon/effect is practically significant. On the flipside, establishing practical significance by looking at how measures correspond to day-to-day behaviors and other meaningful outcomes can be a strong validation strategy (Blanton \& Jaccard, 2006)

For example, certain variants of the implicit association test (IAT) procedures might truly capture implicit attitudes, however if the connection between implicit attitudes and prejudicial behaviors are never inquired, then the valid tool cannot be determined to be practical.

\footnotetext{
${ }^{4}$ Effect size benchmarks can also be used to aid in statistical power analysis (Paterson, Harms ,Steel \& Crede, 2015), in creating non-nil predictions for stronger theoretical refinement (Bosco et al.,
}

Externally validity isn't also necessarily practically significant. An independent variable might affect the dependent variable (as in attitude measured through Likert type scales) in the lab which then replicates to a community setting, but this causal link may not extend towards other practical outcomes i.e prejudicial attitudes might be lessened, but actual behaviors and local policy voting preference might not change. That is, it may be externally valid, but not externally practical.

Practically significant is not necessarily theoretical significant. It may be good at this point to reiterate that most studies in social psychology may be designed for theoretical significance - to contribute to basic knowledge of human behavior, affect and cognitionand practical significance and application is a secondary concern. Although many fail to achieve this goal (Simmons et al., 2011; Antonakis, 2017; Chambers, 2017). Whether any given study/program of study is theoretically significant depends on the extent that it rigorously advances knowledge and answers important questions, which among many others include: what does the data imply of theory, how do we select between competing models, and when are hypotheses and theories falsified?

What is theoretically significant and meaningful is intimately linked with good science which is dealt under the fields of philosophy of science (e.g. Dienes, 2008, Chalmers, 2013) and philosophy of statistics (e.g. Mayo, 2018; Gelman \& Shalizi, 2013; McElreath 2016). At the heart of inference is the question of what can be inferred from observed data, and what can't; and establishing theoretically significant studies may be more difficult than establishing practically significant ones. There are many studies that are of great theoretical significance, but with no immediate practical significance. However, whether basic or applied, "research that is not rigorous simply cannot be relevant" (Antonakis, 2017; Vermeulen, 2005)

However, there is no reason to deny that both are tightly coupled. (Steg \& Rothengatter, 2008) Theory allows us to meaningfully direct our efforts and allow us to extrapolate what and when practical outcomes can happen and under what conditions. (Rothman, 2004) And practical outcomes, through various processes such as the generation of novel hypotheses (Schultz \& Estrada-Hollenbeck, 2008; Steg \& Rothengatter, 2008) and the testing of setting dependence of causal relations

2015) as well as helping indicate where more research would be beneficial (Colquitt \& Zapata-Phelan, 2007) 
help refine theory ${ }^{5}$ (Rothman, 2004). Both theory and practice set proper benchmarks for the meaningful interpretation of effect sizes (Orlitzky, 2014)

The practical significance approach -without a strong theory- can be piecemeal, largely exploratory and 'black box' (or that explanatory mechanisms or processes are lower in priority rather than whether the outcomes themselves are relevant). However, in the spirit of pragmatism, a main advantage is that even if we fail in a lot of ways over theoretical matters -theories will change and refine, models can be broken tested and replaced, correlational and quasi-experimental studies may be found to be confounded- in the moment we conduct our study with practical significance approach in mind, we set the ground to make a difference and affect change.

That is, we ask research psychologists, despite tragic incentive structures, despite convenience and numerous deterrents, despite the difficulty of human science, to go back into the heart of why we chose this profession: to matter

\section{SOLUTIONS, DIRECTIONS AND EXAMPLES}

\section{FIELD EXPERIMENTS}

Field experiments are "randomized studies that are conducted in real-world settings" (Gerber \& Green, 2012). A major benefit of field experiments, when done well, is that it allows for strong causal inference and external validity, if not generalizability (Eden, 2017). And this is especially contrasted with laboratory research, and common self-report/survey correlational research. While often overlooked as a design option, it produces actionable knowledge (Eden, 2017) because it reveals whether causal relationships and interventions hold up in the 'real world' despite a multitude of social influences (Paluck \& Cialdini, 2014). While laboratory experiments isolate and inquire what can happen, field experiments directly examine whether this does happen (Mook, 1983). And while correlational research serve important functions, these seem to be often done out of convenience, with little regard for foundational measurement considerations, like validity (Flake, Pek \& Hehman, 2017) and other issues, including endogeneity (Antonakis, 2017)

More importantly, because field experiments direct interventions to individuals/units of interest in their natural settings, measured outcomes are almost always what makes sense in their context - and thus of the

\footnotetext{
${ }^{5}$ Bronfenbrenner (1997) reverses the famous Lewin maxim and writes that "There is nothing like the practical to build a good theory"
}

practical variety. While there are challenges, including those of implementation logistics, coordination and analysis (Eden, 2017; Gerber \& Green, 2012) the advantages of a field experiment for both practical significance and theory advancement should put it as a top consideration for any research inquiry.

Inspiring examples include Paluck's (2009) field experiment, testing whether mass media - through a soap opera that is modelling reconciliatory behaviors affect intergroup prejudice, cooperation and social norms in post-genocide Rwanda. Blattman, Jamison \& Sheridan (2015) meanwhile show that cognitive behavioral therapy (CBT) with cash grants reduce crime and violence even 1 year later compared to interventions with cash alone or CBT alone. Their field experiment in Liberia also show that due to the therapy sessions, the participants' identities changed from regarding themselves as being ostracized to being mainstream members of the community. Broockman \& Kalla (2016), in a well-powered field experiment show that a 10 minute face-to-face conversation with a mixture of deliberate and effortful processing as well as perspective-taking (imagining another person's point of view) durably reduces negative attitude towards trans individuals even after 3 months.

\section{THE USE OF NON-ARBITRARY METRICS AND USE OF MIXED-METHODS}

Much of psychology deals with hard to observe variables, including attitudes, beliefs, emotions, prejudice etc. What is often done is to measure these using a Likert type scale, and finding the metric corresponding to an individual - that is the "individuals' standings on the construct of interest" (Blanton \& Jaccard, 2006) arbitrary metrics are when one cannot be sure of the correspondence between the metric and the true location of the individual in the underlying dimension of a variable. For example if 7 items all purporting to measure 'self-esteem' have a 5 point anchor, an average score of 4.71 is arbitrary). The total sum of the items, 33, also give the same inference for many analyses using standard linear models and assuming classic scoring procedures; but 4.71 and 33 obviously cannot both be located in the same spot of the underlying dimension of selfesteem; the mapping is arbitrary. Very often, how these scores gain interpretation is only through the comparison of the scores of other individuals

However, some quantitative metrics are immediately meaningful: metrics in outcomes such as divorce 
(even 'transforming' to numbers 0 for married, 1 for divorce), mortality, vote in a particular policy, absenteeism, frequency of violent crimes and other systematic observation of behaviors are all non-arbitrary in the sense that they do not need a correspondence to underlying dimension or variable to make sense. These can be used as indicators for a speculated latent variable, say "irresponsibility" in which case the problems discussed above resurface, but by the themselves they stand on their own. Likewise, many information that is accessed by qualitative approaches (including systematic, participant and naturalistic observations) can be meaningful on their own, or as building blocks for inference for a latent variable. But it bears emphasis that naturalistic observation of human behavior is a large class of non-arbitrary measures.

Measurement will always be a foundational challenge for psychology (Borsboom, 2005; Flake, Pek, \& Hehman, 2017; Bandalos, 2018) and the field of psychometrics is poised to tackle these challenges. However, in the absence of strong and credible programs of measurement (e.g. Benson, 1998) in a particular research inquiry, pragmatic researchers are urged to use both well-measured but arbitrary metrics with non-arbitrary ones: that is to sample a range of outcomes to triangulate the importance and consequences of an effect. And these include the combination of both qualitative and quantitative approaches (dubbed as 'mixedmethods') the information of both will help in the evaluation of a study's practical significance.

Not only are qualitative approaches relevant and useful in their own right but they also help in understanding the numerical indices of quantitative approaches, whether by offering plausible mechanisms for the causal effect (Paluck, 2010) or enriching the observed outcomes to include the behaviors, interactions as well as contexts of the participants' responses. Further, qualitative information is one of the ways to 'audit' p-values and statistical assumptions in error statistics (Mayo, 2018) that help us quantify whether our claim, given the data at hand, is warranted. In particular, culture sensitive 'indigenous' methods are needed to understand local phenomena and sense-making with fidelity (Pe-Pua, 2006) but sound analysis still depend on the rigor of the qualitative approach (Toma, 2011; Moravcsik, 2010)

Ethnographic and other qualitative approaches embedded in a field experiment is also a promising combination (Paluck, 2010) with one form being "experimental ethnography" where effects of randomized treatments to all units/individuals are ethnographically observed instead of being measured or summarized with numerical indices.

Example of studies with a strong qualitative component include a study by den Nieuwenboer, Cunha, \& Treviño, (2017) where using participant observation and other ethnographic methods in a 15-month data gathering period, the authors were able trace how in a particular company, directives from upper management gets 'translated' into deceptive performance in lower levels (such as in false performance and sales data) because of middle managers' deliberate exploitation of routines. All three examples of the field experiments discussed above also have a qualitative component, making their observations of the phenomena 'thicker' and better contextualized.

Examples of studies that use non-arbitrary metrics, but are nonetheless not qualitative in nature include those of campaigning to vote studies- looking at whether certain campaign tactics increase voting turnout (Green \& Gerber, 2015) and whether more policing reduces crime for an area, or merely displaces these to nearby areas (Blattman, Green, Ortega \& Tobón, 2018). Meanwhile, Antonakis, d'Adda, Weber, \& Zehnder (2015) test in an organizational field experiment whether the use of charismatic leadership tactics increase the actual financial output of a firm through increased worker effort/productivity.

\section{DAISY CHAIN / CUMULATIVE ENTERPRISE / STRONG RESEARCH PROGRAM}

Experiments in psychology seek to isolate phenomena -about human behavior, cognition and affect- and understand its mechanisms. However, if field experiments aren't used, how mechanisms in the lab correspond to practical "real world" outcomes very often is not made in any single study, but requires a 'daisy chain', a series of linked studies. It may be found in the laboratory that an effect exists (such as stereotype threat- where awareness of a person's potential negative stereotype affects his/her performance; Spencer, Steele \& Quinn, 1999) but later research that tries to link this with ecological support might fail to find support that the mechanism underlies the observed phenomena (e.g. Stafford, 2018)

A strong research program is one of the few viable ways that we 'learn to make errors talk' (Mayo, 1996). In particular, for results and effect sizes that fit differing models and theories, strong research programs allow for ruling out competing explanations, sifting signal from noise, and partitioning the responsibility of core vs auxiliary factors in producing the results found in studies. 
These coherent series of linked studies provide some of the strongest contributions of psychology. Before consequential entertainment-education radio dramas such as Tanzania's "Twende na Wakati," or "Let's Go with the Times," were able to increase country-wide family planning behavior, including the increased use contraception and awareness of HIV/AIDS prevention (Rogers et al., 1999; Smith, 2002) through modelling desired behaviors and being a source of self-efficacy for these new behaviors, Bandura's social learning theory in itself must already have been thoroughly examined (see also: Singhal, Cody \& Rogers, 2003; Singhal \& Rogers, 2012 for a review of entertainment-educational radio soap operas for behavioral change across diverse settings including India, Peru, South Africa and many others). Bandura's work represents a strong research program, albeit more confirmationist, with decades of cumulative work (Bandura, 2012) starting from the late 1950's; tackling wide but interconnected constructs such as modelling and self-efficacy with a broad range of applicability, informing variants of behavioral therapy in clinical psychology, effects of mass media, education and many others. (Although see Yeo \& Neal, 2013; Vancouver, 2012; Bandura, 2015; Vancouver \& Purl, 2017 for debates on the dynamics of self-efficacy)

Other strong research programs include Milgram's obedience studies, where 17 different variations of the original electric shock - learning situation were conducted (Burger, 2017) over the span of almost a decade. Variations include the modification of the characteristics of the authority figure, proximity of the parties involved, and other situational variables. That a portion of individuals 'obey' perceived authorities even for clearly harmful acts is unfortunately also replicable across cultures; with the basic procedure being replicated in 9 different countries: Italy, South Africa, Germany, Australia, Jordan, Spain, India, Austria and Poland (Blass, 2012; Doliński et al., 2017). Although this lineage of work has traditionally been more theoretically generative (for example, in explaining why people obey; Reicher, Haslam \& Smith, 2012; Burger, 2017) rather than definitively establishing how it affects participants' further day-to-day behaviors and other outcomes.

\section{ASSUMPTIONS \& DESIDERATA}

Any one study, especially with small sample size, noisy and poorly validated measures, inaccessible data and mindlessly analyzed, practical significance will always be hard to establish. That is, for a number of published articles in the field, which do not meet the standards above (Simmons et al., 2011; Antonakis, 2017;
Chambers, 2017; Flake, Pek, \& Hehman, 2017) practical significance is (sadly) undeterminable. Thus the discussion above assumes rigor in any single study, and that major threats to inference are addressed: there is measurement validity, measurement reliability, statistical and model assumptions are met, assumptions of pooling in meta-analysis are tenable, the results are robust across sensitivity/multiverse analyses, and is replicable and generalizable across relevant moderators (contexts, units etc.)

Further, our jumping point here for practical significance are effect sizes, but these are not without its critics. First, effect sizes must be chosen carefully (Pintea, 2010; Ferguson, 2009; Kelley \& Preacher, 2002) and interpreted judiciously, which may otherwise mislead researchers into thinking an effect size answers a question it doesn't (e.g for $\mathrm{r}^{2}$ see Ford, 2015; Shalizi, 2015). Beyond this is the consideration of the philosophical basis of effect sizes (Simonsohn, 2015). An effect size at its essence is the "the amount of something that might be of interest." (Cumming, 2013). This is estimated from a sample, with bias correction for generalizing to some actual or conceptual population. However, take for example, a straightforward effect size: the mean difference between two groups. Does any process, say intergoup contact hypothesis or the anchoring effect, have any "true" mean difference effect size estimate? The obvious answer seems no. The effect whether as difference between groups or strength of relationships- is a function not just of the underlying phenomena itself, but also of the study design, of the type of stimulus, of the IV's observed/manipulated 'dosage', of within group variability, of other predictors in the model et cetera. Effect sizes then, are approximates of what we expect to happen under particular conditions, and this estimation is produced by and filtered through a model of our choosing. There is no free lunch for non-mindless statistics. Even estimation thinking and the practical significance that interprets and extends it- needs good judgement. 


\section{References}

Aguinis, H., Werner, S., Lanza Abbott, J., Angert, C., Park, J. H., \& Kohlhausen, D. (2010). Customer-centric science: Reporting significant research results with rigor, relevance, and practical impact in mind. Organizational Research Methods, 13(3), 515539.

Antonakis, J. (2017). On doing better science: From thrill of discovery to policy implications. The Leadership Quarterly, 28(1), $5-21$.

Antonakis, J., d'Adda, G., Weber, R., \& Zehnder, C. (2015). "Just words? Just speeches?": On the economic value of charismatic leadership. Retrieved from: www.hec.unil.ch/jantonakis/ADWZ_Charisma_Nov2015.pdf

Aronow, P. M., \& Samii, C. (2013). Estimating Average Causal Effects Under General Interference, with Application to a Social Network Experiment. arXiv preprint arXiv:1305.6156.

Baguley, T. (2012). Serious stats: A guide to advanced statistics for the behavioral sciences. Macmillan International Higher Education.

Baldwin, S. A., Imel, Z. E., Braithwaite, S. R., \& Atkins, D. C. (2014). Analyzing multiple outcomes in clinical research using multivariate multilevel models. Journal of consulting and clinical psychology, 82(5), 920.

Banas, J. A., \& Rains, S. A. (2010). A meta-analysis of research on inoculation theory. Communication Monographs, 77(3), 281311.

Bandalos, D. L. (2018). Measurement theory and applications for the social sciences, pp 254-296

Bandura, A. (2012). Social cognitive theory. Theories of social psychology: an introduction. In van Lange, Kruglanski \& Higgins, eds, Handbook of Theories of Social Psychology, vol. 1. London: Sage Publications.

Benartzi, S., Beshears, J., Milkman, K. L., Sunstein, C. R., Thaler, R. H., Shankar, M., ... \& Galing, S. (2017). Should governments invest more in nudging?. Psychological science, 28(8), 10411055.

Benson, J. (1998). Developing a strong program of construct validation: A test anxiety example. Educational Measurement: Issues and Practice, 17(1), 10-17.

Blanton, H., \& Jaccard, J. (2006). Arbitrary metrics in psychology. American Psychologist, 61(1), 27.

Blattman, C., Green, D., Ortega, D., \& Tobón, S. (2018). Hotspots interventions at scale: the direct and spillover effects of policing and city services on crime in Bogotá. Retrieved from: http://www.3ieimpact.org/media/filer_public/2018/10/09/gfrdpw11044-hotspots-interventions-scale.pdf

Blattman, C., Jamison, J. C., \& Sheridan, M. (2015). Reducing crime and violence: Experimental evidence from cognitive behavioral therapy in Liberia(No. w21204). National Bureau of Economic Research

Borsboom, D. (2005). Measuring the mind: Conceptual issues in contemporary psychometrics. Cambridge University Press.

Borsboom, D. (2013). Theoretical amnesia. Retrieved from: osc.centerforopenscience.org/2013/11/20/theoretical-amnesia/. Mirror: https://web.archive.org/web/20181208115425/http://osc.centerforopenscience.org/2013/11/20/theoretical-amnesia/

Borsboom, D. (2017). A network theory of mental disorders. World psychiatry, 16(1), 5-13.
Borsboom, D., Kievit, R. A., Cervone, D., \& Hood, S. B. (2009). The two disciplines of scientific psychology, or: The disunity of psychology as a working hypothesis. In Dynamic process methodology in the social and developmental sciences (pp. 67-97). Springer, New York, NY.

Bosco, F. A., Aguinis, H., Singh, K., Field, J. G., \& Pierce, C. A. (2015). Correlational effect size benchmarks. Journal of Applied Psychology, 100(2), 431.

Brand, J. E., \& Halaby, C. N. (2006). Regression and matching estimates of the effects of elite college attendance on educational and career achievement. Social Science Research, 35(3), 749770.

Bronfenbrenner, U. (1977). Lewinian Space and Ecological Substance. Journal of Social Issues, 33(4), 199-212. doi:10.1111/j.1540-4560.1977.tb02533.x

Broockman, D., \& Kalla, J. (2016). Durably reducing transphobia: A field experiment on door-to-door canvassing. Science, 352(6282), 220-224.

Burger, J (2017).Obedience. In Harkins, S. G., Williams, K. D., \& Burger, J. M. (Eds.) The Oxford handbook of social influence, 317.

Butera, F., Falomir-Pichastor, J. M., Mugny, G., \& Quiamzade, A. (2017). Minority influence. The Oxford handbook of social influence, 317-337.

Cabrera, E. F., \& Raju, N. S. (2001). Utility analysis: Current trends and future directions. International Journal of Selection and Assessment, 9(1-2), 92-102.

Chalmers, A. F. (2013). What is this thing called science?. Hackett Publishing.

Chambers, C. (2017). The seven deadly sins of psychology: A manifesto for reforming the culture of scientific practice. Princeton University Press.

Chan, C. H., \& Fu, K. W. (2015, June). Predicting Political Polarization from Cyberbalkanization: Time series analysis of Facebook pages and Opinion Poll during the Hong Kong Occupy Movement. In Proceedings of the ACM Web Science Conference (p. 36). ACM.

Chan, C. H., \& Fu, K. W. (2015, June). Predicting Political Polarization from Cyberbalkanization: Time series analysis of Facebook pages and Opinion Poll during the Hong Kong Occupy Movement. In Proceedings of the ACM Web Science Conference (p. 36). ACM.

Cohen, J. (1962). The statistical power of abnormal-social psychological research: A review. The Journal of Abnormal and Social Psychology, 65, 145-153. doi:10.1037/h0045186

Cohen, J. (1988). Statistical power analysis for the behavioral sciences. (2nd ed.). Hillsdale, NJ: Erlbaum.

Cook, R. J., \& Sackett, D. L. (1995). The number needed to treat: a clinically useful measure of treatment effect. BMJ: British Medical Journal, 310(6977), 452.

Cortina, J. M., \& Landis, R. S. (2009). When small effect sizes tell a big story, and when large effect sizes don't. Statistical and methodological myths and urban legends: Doctrine, verity and fable in the organizational and social sciences, 287-308.

Cramer, A. O., Van der Sluis, S., Noordhof, A., Wichers, M., Geschwind, N., Aggen, S. H., ... \& Borsboom, D. (2012). Dimensions of normal personality as networks in search of equilibrium: You can't like parties if you don't like people. European Journal of Personality, 26(4), 414-431.

Credé, M. (2018). A Negative Effect of a Contractive Pose is Not Evidence for the Positive Effect of an Expansive Pose: Commentary on Cuddy, Schultz, and Fosse (2018). 
Credé, M., \& Phillips, L. A. (2017). Revisiting the Power Pose Effect: How Robust Are the Results Reported by Carney, Cuddy, and Yap (2010) to Data Analytic Decisions?. Social Psychological and Personality Science, 8(5), 493-499.

Cuddy, A. J., Schultz, S. J., \& Fosse, N. E. (2018). P-Curving a More Comprehensive Body of Research on Postural Feedback Reveals Clear Evidential Value For Power-Posing Effects: Reply to Simmons and Simonsohn (2017). Psychological science, 29(4), 656-666.

Cumming, G. (2013). Understanding the new statistics: Effect sizes, confidence intervals, and meta-analysis. Routledge.

Cumming, G. (2014). The new statistics: Why and how. Psychological science, 25(1), 7-29.

Cunningham, S. (2018). Causal inference: The mixtape (V.17). Retrieved from: https://docmimic.com/l-HKrn4Nd/causal-inference-the-mixtape.html

den Nieuwenboer, N. A., Cunha, J. V. D., \& Treviño, L. K. (2017). Middle managers and corruptive routine translation: the social production of deceptive performance. Organization Science, 28(5), 781-803.

Deslauriers, L., McCarty, L. S., Miller, K., Callaghan, K., \& Kestin, G. (2019). Measuring actual learning versus feeling of learning in response to being actively engaged in the classroom. Proceedings of the National Academy of Sciences, 201821936.

Dienes, Z. (2008). Understanding psychology as a science: An introduction to scientific and statistical inference. Macmillan International Higher Education.

Ditlmann, R., \& Paluck, E. L. (2015). Field experiments. International Encyclopedia of the Social and Behavioral Sciences, 9, 128-34.

Doliński, D., Grzyb, T., Folwarczny, M., Grzybała, P., Krzyszycha, K., Martynowska, K., \& Trojanowski, J. (2017). Would You Deliver an Electric Shock in 2015? Obedience in the Experimental Paradigm Developed by Stanley Milgram in the 50 Years Following the Original Studies. Social Psychological and Personality Science, 8(8), 927-933. doi:10.1177/1948550617693060

Eckles, D., Karrer, B., \& Ugander, J. (2017). Design and analysis of experiments in networks: Reducing bias from interference. Journal of Causal Inference, 5(1).

Eden, D. (2017). Field experiments in organizations. Annual Review of Organizational Psychology and Organizational Behavior, 4, 91-122.

Eliot, L. (2010). Pink brain, blue brain. Oneworld Publications.

Ellis, S. M., \& Steyn, H. S. (2003). Practical significance (effect sizes) versus or in combination with statistical significance (pvalues): research note. Management Dynamics: Journal of the Southern African Institute for Management Scientists, 12(4), 5153.

Ferguson, C. J. (2009). An effect size primer: A guide for clinicians and researchers. Professional Psychology: Research and Practice, 40(5), 532.

Fiedler, K. (2018). The creative cycle and the growth of psychological science. Perspectives on Psychological Science, 13(4), 433438.

Fisher, A. J., Medaglia, J. D., \& Jeronimus, B. F. (2018). Lack of group-to-individual generalizability is a threat to human subjects research. Proceedings of the National Academy of Sciences, 27 https://doi.org/10.1073/pnas.1711978115

Flake, J. K., Pek, J., \& Hehman, E. (2017). Construct validation in social and personality research: Current practice and recommendations. Social Psychological and Personality Science, 8(4), 370-378.
Ford, C. (2015, October 17). Is R-squared useless?. Retrieved from: https://data.library.virginia.edu/is-r-squared-useless/ Mirror: https://web.archive.org/web/20190831232438/https://data.library.virginia.edu/is-r-squared-useless/

Frese, M., Gielnik, M. M., \& Mensmann, M. (2016). Psychological training for entrepreneurs to take action: Contributing to poverty reduction in developing countries. Current Directions in Psychological Science, 25(3), 196-202.

Fried, E. I. (2017). What are psychological constructs? On the nature and statistical modelling of emotions, intelligence, personality traits and mental disorders. Health psychology review, 11(2), 130-134.

Gelman, A., \& Shalizi, C. R. (2013). Philosophy and the practice of Bayesian statistics. British Journal of Mathematical and Statistical Psychology, 66(1), 8-38.

Gerber, A. S., \& Green, D. P. (2012). Field experiments: Design, analysis, and interpretation. WW Norton.

Gidengil, E., Wass, H., \& Valaste, M. (2016). Political Socialization and Voting: The Parent-Child Link in Turnout. Political Research Quarterly, 69(2), 373-383.

Giuffrida, A., \& Torgerson, D. J. (1997). Should we pay the patient? Review of financial incentives to enhance patient compliance. Bmj, 315(7110), 703-707.

Gomes, C. M. A., de Araujo, J., do Nascimento, E., \& Jelihovschi, E. G. (2018). Routine Psychological Testing of the Individual Is Not Valid. Psychological reports, 0033294118785636.

Green, D. P., \& Gerber, A. S. (2015). Get out the vote: How to increase voter turnout. Brookings Institution Press.

Grissom, R. J., \& Kim, J. J. (2005). Effect sizes for research: A broad practical approach. Lawrence Erlbaum Associates Publishers.

Guyatt, G. H., Osoba, D., Wu, A. W., Wyrwich, K. W., Norman, G. R., \& Clinical Significance Consensus Meeting Group. (20021). Methods to explain the clinical significance of health status measures. In Mayo Clinic Proceedings (Vol. 77, No. 4, pp. 371383). Elsevier.

Guyatt, G., Rennie, D., Meade, M., \& Cook, D. (Eds.). (2002). Users' guides to the medical literature: a manual for evidence-based clinical practice (Vol. 706). Chicago: AMA press.

Hoekstra, M. (2009). The effect of attending the flagship state university on earnings: A discontinuity-based approach. The Review of Economics and Statistics, 91(4), 717-724.

Imai, K., \& Ratkovic, M. (2013). Estimating treatment effect heterogeneity in randomized program evaluation. The Annals of Applied Statistics, 7(1), 443-470.

Jacobson, N. S., \& Truax, P. (1991). Clinical significance: a statistical approach to defining meaningful change in psychotherapy research. Journal of consulting and clinical psychology, 59(1), 12.

Johnson, G., Lewis, R. A., \& Reiley, D. (2016). Location, location, location: repetition and proximity increase advertising effectiveness. http://dx.doi.org/10.2139/ssrn.2268215

Kelley, K., \& Preacher, K. J. (2012). On effect size. Psychological methods, 17(2), 137.

Kirk, R. E. (1996). Practical significance: A concept whose time has come. Educational and psychological measurement, 56(5), 746-759.

Klein, R. A., Vianello, M., Hasselman, F., Adams, B. G., Adams Jr, R. B., Alper, S., ... \& Batra, R. (2018). Many Labs 2: Investigating variation in replicability across samples and settings. Advances in Methods and Practices in Psychological Science, 1(4), 443-490.

Kline, R. B. (2004). Beyond significance testing: Reforming data analysis methods in behavioral research. 
Kline, R. B. (2008). Becoming a behavioral science researcher: A guide to producing research that matters. Guilford Press.

Kruschke, J. (2014). Doing Bayesian data analysis: A tutorial with R, JAGS, and Stan. Academic Press.

Kulik, C. T., \& Roberson, L. (2008). 8 Diversity initiative effectiveness: What organizations can (and cannot) expect from diversity recruitment, diversity training, and formal mentoring programs. Diversity at work, 265-317.

Lakens, D. (2016) Improving your statistical inferences. Accessed through: https://www.coursera.org/learn/statistical-inferences

Lakens, D. (2017). Equivalence tests: a practical primer for t tests, correlations, and meta-analyses. Social Psychological and Personality Science, 8(4), 355-362.

Lambert, M. J., Hansen, N. B., \& Bauer, S. (2008). Assessing the clinical significance of outcome results.

LeBaron, A. B., Hill, E. J., Rosa, C. M., \& Marks, L. D. (2018). Whats and hows of family financial socialization: Retrospective reports of emerging adults, parents, and grandparents. Family Relations, 67(4), 497-509.

Leech, N. L., \& Onwuegbuzie, A. J. (2004). A proposed fourth measure of significance: The role of economic significance in educational research. Evaluation \& Research in Education, 18(3), 179-198.

Lewis, C.W., Cobb, R.B., Winokur, M., Leech, N., Viney, M. and White, W. (2003) The effects of full and alternative day block scheduling on language arts and science achievement in a junior high school.

Lydick, E., \& Epstein, R. S. (1993). Interpretation of quality of life changes. Quality of life Research, 2(3), 221-226.

Matz, S. C., Gladstone, J. J., \& Stillwell, D. (2017). In a world of big data, small effects can still matter: a reply to Boyce, Daly, Hounkpatin, and Wood (2017). Psychological science, 28(4), 547-550.

Mayo, D. G. (1996). Error and the growth of experimental knowledge. University of Chicago Press.

Mayo, D. G. (2018). Statistical inference as severe testing: How to get beyond the statistics wars. Cambridge University Press.

McElreath, R. (2016). Statistical Rethinking: A Bayesian Course with Examples in R and Stan (Vol. 122). CRC Press.

McGlinchey, J. B., Atkins, D. C., \& Jacobson, N. S. (2002). Clinical significance methods: Which one to use and how useful are they?. Behavior Therapy, 33(4), 529-550.

McGuire, W. J. (1964). Inducing resistance to persuasion: Some contemporary approaches. In L. Berkowitz (Ed.), Advances in experimental social psychology (Vol. 1, pp. 191_229). New York, NY: Academic Press.

Mohr, D. C., Hart, S. L., Howard, I., Julian, L., Vella, L., Catledge, C., \& Feldman, M. D. (2006). Barriers to psychotherapy among depressed and nondepressed primary care patients. Annals of Behavioral Medicine, 32(3), 254-258.

Mook, D. G. (1983). In defense of external invalidity. American psychologist, 38(4), 379.

Moravcsik, A. (2010). Active citation: A precondition for replicable qualitative research. PS: Political Science \& Politics, 43(1), 2935.

Moshontz, H., Campbell, L., Ebersole, C. R., IJzerman, H., Urry, H. L., Forscher, P. S., ... \& Castille, C. M. (2018). The psychological science accelerator: Advancing psychology through a distributed collaborative network. Advances in Methods and Practices in Psychological Science, 1(4), 501-515.

Munafò, M. R., Nosek, B. A., Bishop, D. V., Button, K. S., Chambers, C. D., Du Sert, N. P., ... \& Ioannidis, J. P. (2017). A manifesto for reproducible science. Nature Human Behaviour, 1(1), 0021.
Neisser, U. (1976). Cognition and reality. Principles and implication of cognitive psychology. San Francisko: WH Freeman and Company.

Nock, M. K., \& Ferriter, C. (2005). Parent management of attendance and adherence in child and adolescent therapy: A conceptual and empirical review. Clinical child and family psychology review, 8(2), 149-166.

Onwuegbuzie, A. J., \& Levin, J. R. (2003). Without supporting statistical evidence, where would reported measures of substantive importance lead? To no good effect. Journal of Modern Applied Statistical Methods, 2(1), 12.

Orlitzky, M. (2014). The new statistics require new social and organization theories. (Unpublished dissertation). The University of South Australia

Osoba, D., Rodrigues, G., Myles, J., Zee, B., \& Pater, J. (1998). Interpreting the significance of changes in health-related qualityof-life scores. Journal of clinical oncology, 16(1), 139-144.

Paluck, E. (2010). The promising integration of qualitative methods and field experiments. The ANNALS of the American Academy of Political and Social Science, 628(1), 59-71.

Paluck, E. L. (2009). Reducing intergroup prejudice and conflict using the media: a field experiment in Rwanda. Journal of personality and social psychology, 96(3), 574.

Paluck, E. L., \& Cialdini, R. B. (2014). Field research methods. Handbook of research methods in social and personality psychology, 81-97.

Paluck, E. L., Shepherd, H., \& Aronow, P. M. (2016). Changing climates of conflict: A social network experiment in 56 schools. Proceedings of the National Academy of Sciences, 113(3), 566571.

Pechmann, C., \& Stewart, D. W. (1988). Advertising repetition: A critical review of wearin and wearout. Current issues and research in advertising, 11(1-2), 285-329.

Pe-Pua, R. (2006). From decolonizing psychology to the development of a cross-indigenous perspective in methodology. In Indigenous and cultural psychology (pp. 109-137). Springer, Boston, MA.

Pichert, D., \& Katsikopoulos, K. V. (2008). Green defaults: Information presentation and pro-environmental behaviour. Journal of Environmental Psychology, 28(1), 63-73.

Pintea, S. (2010). The relevance of results in clinical research: Statistical, practical, and clinical significance. Journal of EvidenceBased Psychotherapies, 10(1), 101.

Plonsky, L., \& Oswald, F. L. (2014). How big is "big"? Interpreting effect sizes in L2 research. Language Learning, 64(4), 878-912.

Prentice, D. A., \& Miller, D. T. (1992). When small effects are impressive. Psychological bulletin, 112(1), 160.

Reicher, S. D., Haslam, S. A., \& Smith, J. R. (2012). Working toward the experimenter: Reconceptualizing obedience within the Milgram paradigm as identification-based followership. Perspectives on Psychological Science, 7(4), 315-324.

Roberts, B. W., Kuncel, N. R., Shiner, R., Caspi, A., \& Goldberg, L. R. (2007). The power of personality: The comparative validity of personality traits, socioeconomic status, and cognitive ability for predicting important life outcomes. Perspectives on Psychological science, 2(4), 313-345.

Rogers, E. M., Vaughan, P. W., Swalehe, R. M., Rao, N., Svenkerud, P., \& Sood, S. (1999). Effects of an entertainment-education radio soap opera on family planning behavior in Tanzania. Studies in family planning, 30(3), 193-211. 
Rothman, A. J. (2004). " Is there nothing more practical than a good theory?": Why innovations and advances in health behavior change will arise if interventions are used to test and refine theory. International Journal of Behavioral Nutrition and Physical Activity, 1(1), 11.

Rumsey, M. G. (2012). Military selection and classification in the United States. The Oxford handbook of military psychology, 129-148.

Schultz, W., \& Estrada-Hollenbeck, M. (2008). The USE of theory in applied social psychology. In Steg, L., Buunk, A.P., \& Rothengatter, T., (Eds.), Applied social psychology: Understanding and managing social problems (pp. 28-49). New York: Cambridge University Press.

Shalizi, C. (2015, October 16). Lecture 10: F-Tests, R2, and Other Distractions. Retrieved from: www.stat.cmu.edu/ cshalizi/mreg/15/lectures/10/lecture-10.pdf Mirror: https://web.archive.org/web/20181123200252/http://www.stat.cmu.edu/ csh alizi/mreg/15/lectures/10/lecture-10.pdf

Shalizi, C. R., \& Thomas, A. C. (2011). Homophily and contagion are generically confounded in observational social network studies. Sociological methods \& research, 40(2), 211-239.

Simmons, J. P., Nelson, L. D., \& Simonsohn, U. (2011). False-positive psychology: Undisclosed flexibility in data collection and analysis allows presenting anything as significant. Psychological science, 22(11), 1359-1366.

Simonsohn, U. (2015). "The" effect size does not exist. Retrieved from: datacolada.org/33. Mirror: https://web.archive.org/web/20190112172226/http://datacolada.org/33

Singhal, A., \& Rogers, E. (2012). Entertainment-education: A communication strategy for social change. Routledge.

Singhal, A., Cody, M. J., Rogers, E. M., \& Sabido, M. (Eds.). (2003). Entertainment-education and social change: History, research, and practice. Routledge.

Smith, D. (2002). The theory heard'round the world. Monitor on psychology, 33(9), 30-32.

Spencer, S. J., Steele, C. M., \& Quinn, D. M. (1999). Stereotype threat and women's math performance. Journal of experimental social psychology, 35(1), 4-28.

Stafford, T. (2018). Female chess players outperform expectations when playing men. Psychological science, 29(3), 429-436.

Steg, L. \& Rothengatter, T. (2008). Introduction to applied social psychology. In Steg, L., Buunk, A.P., \& Rothengatter, T., (Eds.), Applied social psychology: Understanding and managing social problems (pp. 1-24). New York: Cambridge University Press.

Steyn, H. S. (2000). Practical significance of the difference in means. SA journal of industrial psychology, 26(3), 1-3.

Stoddart, K. P. (1999). Adolescents with Asperger syndrome: Three case studies of individual and family therapy. Autism, 3(3), 255271.

Szapocznik, J., \& Williams, R. A. (2000). Brief strategic family therapy: Twenty-five years of interplay among theory, research and practice in adolescent behavior problems and drug abuse. Clinical child and family psychology review, 3(2), 117-134.

Thompson, B. (2002). "Statistical,"“practical," and "clinical": How many kinds of significance do counselors need to consider? Journal of Counseling \& Development, 80(1), 64-71.

Toma, J. D. (2011). Approaching rigor in Applied Qualitative Research. The SAGE handbook for research in education, 263.

Vaske, J. J., Gliner, J. \& Morgan, G. (2002). Communicating judgments about practical significance: Effect size, confidence intervals and odds ratios. Human Dimensions of Wildlife, 7(4), 287300 .
Vermeulen, F. (2005). On rigor and relevance: Fostering dialectic progress in management research. Academy of Management Journal, 48(6), 978-982.

Yeaton, W. H., \& Sechrest, L. (1981). Meaningful measures of effect. 